This is a post-peer-review, pre-copyedit version of an article published in Journal of Thermal Analysis and Calorimetry. The final authenticated version is available online at:

https://doi.org/10.1007/s10973-018-7738-1

This version is available from https://hdl.handle.net/10195/74727

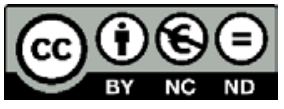

This postprint version is licenced under a Creative Commons Attribution-NonCommercialNoDerivatives 4.0.International. 


\title{
Importance of proper baseline identification for the subsequent kinetic analysis of derivative kinetic data, part 3
}

Roman Svoboda*

Department of Physical Chemistry, Faculty of Chemical Technology, University of Pardubice, Studentska 573, 53210 Pardubice, Czech Republic.

\begin{abstract}
Theoretically simulated kinetic data were used to evaluate the performance of the linear, cubic spline and Bezier mathematic interpolations (in comparison with the physically meaningful tangential area-proportional interpolation) in case of the complex kinetics involving two independent processes with different degrees of overlapping. The Bezier interpolation exhibited best performance; the linear interpolation performed significantly worse than the two other ones. In general, the data-distortions caused by application of the mathematic interpolations do not significantly influence the model-free kinetic parameters apparent activation energy and pre-exponential factor; errors below $2 \%$ occurred even for the most extremely distorted data. On the other hand, the integrated peak area and the modelbased parameters such as kinetic exponents and the complexity ratio can be significantly influenced by the interpolations-caused data distortions, with the associated errors being in the order of tenths of percent. Nevertheless, the distortions associated with the choice of the thermokinetic interpolation were found to not affect the thermal stability predictions for the complex kinetic processes; only the precise predictions based on the mid-range degrees of conversion (e.g. controlled preparation of glass-ceramics) can be significantly affected by the incorrect interpolation of the thermokinetic background.
\end{abstract}

Keywords: baseline, data-distortion, kinetic analysis, complex processes, kinetic prediction

\footnotetext{
* Corresponding author: Tel.: +420 466037346 E-mail address: $\underline{\text { roman.svoboda@ upce.cz }}$
} 


\section{Introduction}

Methodologies-wise, the kinetic treatment of thermal analysis data was during the past years improved almost to perfection. Numerous methods were developed and many equations were derived in order to (more-or-less) accurately determine the quantities from the basic kinetic equation $[1,2]$ :

$$
d \alpha / d t=I \cdot A \cdot e^{-E / R T} \cdot f(\alpha)
$$

where $\alpha$ is the degree of conversion, $t$ is time, $T$ is temperature, $f(\alpha)$ is a substitute for a kinetic model, $I$ is the integrated area under the kinetic peak, $A$ is the pre-exponential factor and $E$ is the apparent activation energy of the process. The most famous model-free methods (being independent from $f(\alpha)$ and designed for determination of $\mathrm{E}$ and $\mathrm{A}$ ) include the methods based on the temperature corresponding to the maximum of the kinetic peak (e.g. Kissinger [3], Ozawa [4], Takhor and Mahadevan [5,6]) and isoconversional methods providing the apparent activation energy in dependence on $\alpha$ (e.g. Friedman [7], Kissinger-Akahira-Sunose [8], Starink [9], Ozawa-Flynn-Wall [10], Vyazovkin [11-13], invariant kinetic parameters method [14]). The model-based methods then involve either non-fitting methods (master plots [15], method based on the compensation effect [16]) or data-fitting methods (combined kinetic analysis [17], Koga method [18], multivariate kinetic analysis [19]). Description and commented analysis of these methods can be found in the Recommendation of ICTAC Kinetic Committee [20]. However, neither the first Recommendation article, nor the following one [21] (aimed at correct data acquisition via the methods of thermal analysis) dealt in detail with proper baseline determination/subtraction in case of the most common, derivative thermokinetic data - usually obtained by differential scanning calorimetry DSC.

The proper subtraction of the thermokinetic background is particularly crucial for kinetic analysis applications because even slight distortion of the data due to the inaccurate baseline determination may lead to significantly different enumeration of the basic kinetic 
equation (Eq. 1) and as a result to largely incorrect kinetic predictions (which are the main goal for applying the kinetic analysis). This was demonstrated recently for the case of single kinetic process [22-24], where the physically meaningful tangential area-proportional (TAP) baseline [25] (see Eq. 2) was replaced by the arbitrary mathematic functions (linear, Bezier [26] or cubic spline baselines [27]) - note that this issue occurs very often in literature (see e.g. [28-32]) because the tangential area-proportional baseline is not implemented in a majority of commercial softwares:

$$
B(T)=(1-\alpha(T)) \cdot\left(z_{0, r}+z_{1, r} \cdot T\right)+\alpha(T) \cdot\left(z_{0, p}+z_{1, p} \cdot\left(T_{f}-T\right)\right)
$$

where $B(T)$ is the temperature dependence of the baseline curve, $\alpha$ is degree of conversion, $z 0, r$ and $z_{1, r}$ are the coefficients characterizing the tangent going through the starting point (in the reactants area), $z_{0, p}$ and $z_{1, p}$ are the coefficients characterizing the tangent going through the end point (in the products area) and $T_{f}$ is the end point temperature. Extensive tests performed on simulated data (and resolving situations involving various peak asymmetries and various heights of the heat capacity steps between the reactants and products) have shown that in most cases the value of activation energy $E$ (and as a consequence also the value of pre-exponential factor A) change only negligibly if the inaccurate subtraction of the thermokinetic background is done. On the other hand, the area of the kinetic peak and the model based parameters, such as the kinetic exponents or the model itself, changed significantly - up to $\Delta \mathrm{I}=30 \%$ and values of kinetic exponents changing by more than $80 \%$ in certain cases.

In the present work we aim to map the situation for the case of complex kinetic processes. Four scenarios covering the most common cases of two-process overlaps will be theoretically simulated with the difference between the heat capacities being modeled by using the TAP baseline. Consequently, the above-mentioned purely mathematical baseline approximations will be applied to subtract the thermokinetic background and detailed kinetic analysis will be applied to demonstrate the deviations arising under the different scenarios. 
The most common sources of errors will be pointed out and discussed with regard to the different types of kinetic complexity.

\section{Data preparation}

The simulations performed to prepare the four datasets were based on the standard kinetic equation (Eq. 1) accommodated for the non-isothermal DSC setup, i.e.:

$$
d \alpha / d t=q^{+} \cdot I \cdot A \cdot e^{-E / R T} \cdot f(\alpha)
$$

where $q^{+}$is the applied heating rate. The complexity of the data is represented by two independent overlapping processes with similar Johnson-Mehl-Avrami (JMA) kinetics [3336] expressed by Eq. 4, where the overlap is adjusted by the pre-exponential factor,

$$
f(\alpha)=n(1-\alpha)[-\ln (1-\alpha)]^{1-(1 / n)}
$$

where $n$ is the model kinetic exponent corresponding to the dimensionality of the process. The overall area under the complex kinetic peak was set to $\mathrm{I}=1$ and the ratio between the subpeak areas corresponding to the two respective sub-processes is equal to $3: 7$. For easier orientation the two sub-peaks will be identified by subscripts $\mathrm{S}\left(=\right.$ small, $\left.\mathrm{I}_{\mathrm{s}} / \mathrm{I}=0.3\right)$ and $\mathrm{L}(=$ large, $\mathrm{I}_{\mathrm{L}} / \mathrm{I}=0.7$ ). The kinetic parameters for one (smaller) peak were: $E_{S}=150 \mathrm{~kJ} \cdot \mathrm{mol}^{-1}$, $A_{S}=10^{15} \mathrm{~s}^{-1}$ and $n_{S}=2$. The kinetic parameters for the other (larger) peak were: $E_{L}=150 \mathrm{~kJ} \cdot \mathrm{mol}^{-1}, n_{L}=2$ and the relative position toward the smaller peak was for each dataset adjusted by changing value of $A_{L}=10^{14} \mathrm{~s}^{-1}, A_{L}=10^{14.5} \mathrm{~s}^{-1}, A_{L}=10^{15.5} \mathrm{~s}^{-1}$ and $A_{L}=10^{16} \mathrm{~s}^{-1}$. Within each dataset the curves for the following $q^{+}$were simulated: $0.5,1,2,5$, 10, 20 and $50{ }^{\circ} \mathrm{C} \cdot \mathrm{min}^{-1}-$ the four respective datasets are depicted in Fig. 1.

For each curve in each dataset two variations (upwards and downwards) of the heat capacity step were prepared by using the TAP baseline. The absolute magnitudes of the heat capacity steps were in all cases similar, equal to $1 \cdot 10^{-4} \mathrm{~d} \alpha / \mathrm{dt}$ step at $q^{+}=0.5^{\circ} \mathrm{C} \cdot \mathrm{min}^{-1}$ (i.e. the thermokinetic backgrounds of the smaller and larger peaks contributed in this case by $3 \cdot 10^{-5}$ 
and $7 \cdot 10^{-5}$, respectively); note that the magnifying effect of $q^{+}$was properly reflected also in the height of the heat capacity steps. Consequently, the arbitrary mathematic functions (linear, Bezier and cubic spline) were used to subtract the TAP-modeled thermokinetic background again - see the two lower graphs in Fig. 1 for the demonstration of the construction of the particular baselines. In this way, 24 datasets containing differently distorted complex kinetic curves were created: 4 types of mutual peak positioning (defined by different $A_{L}$ ) x 3 types of baselines (linear, Bezier, cubic spline) x 2 directions of the heat capacity change (upwards, downwards).

\section{Results of kinetic analysis and discussion}

Kinetic analysis of the distorted complex kinetic datasets was performed by means of model-free and model-based approaches [20]; the following methods were used - modified isoconversional KAS [9] (Eq. 5), isoconversional Friedman [7] (Eq. 6) and multivariate kinetic analysis [19] (Eqs. 7 and 8):

$$
\begin{aligned}
& \ln \left(\frac{q^{+}}{T_{\alpha}^{1.92}}\right)=-1.008 \frac{E}{R T_{\alpha}}+\text { const. } \\
& \ln \left([d \alpha / d t]_{\alpha}\right)=-\frac{E}{R T_{\alpha}}+\text { const. } \\
& R S S=\sum_{j=1}^{n} \sum_{k=\text { First }_{j} i}^{\text {Last }_{j}} w_{j, k}\left(Y \exp _{j, k}-Y c a l_{j, k}\right)^{2} \\
& w_{j}=\frac{1}{\left|[d \alpha / d t]_{\max }\right|_{j}+\left|[d \alpha / d t]_{\min }\right|_{j}}
\end{aligned}
$$

where $(\mathrm{d} \alpha / \mathrm{d} t)_{\alpha}$ and $T_{\alpha}$ are the conversion rate and temperature corresponding to arbitrarily chosen values of conversion $\alpha, R S S$ is the sum of squared residua, $n$ is number of simulated curves, $j$ is index of the given simulated curve, First $t_{j}$ is the index of the first point of the given curve, Last $t_{j}$ is the index of the last point of the given curve, $\operatorname{Yexp}_{j, k}$ is the experimental value 
of the point $k$ of curve $j$, Ycal $_{j, k}$ is the calculated value of the point $k$ of curve $j$ and $w_{j}$ is weighting factor for curve $j$.

Starting with the model-free analysis, in Fig. 2 the resulting E values obtained for all simulated datasets via the modified KAS and Friedman methodologies are shown. For each method two evaluations are shown, averaging the $E$ - $\alpha$ dependences in the $0.1-0.9$ and 0.3 $0.7 \alpha$ ranges, respectively. As is apparent, the range of averaging does not play major role in the determination of the mean $E$ value. The deviations caused by the application of mathematic baselines are generally very small $(\sim 0.7 \%)$ and even in the most extreme cases do not exceed $2 \%$. As expected, the datasets with significantly separated peaks (cases with $A_{L}=10^{14} \mathrm{~s}^{-1}$ and $A_{L}=10^{16} \mathrm{~s}^{-1}$ ) exhibit larger absolute errors (i.e. lower accuracy) and also larger error bars (i.e. lower precision) during the isoconversional evaluation of apparent activation energy. The data also show that the magnitude of errors caused by the usage of the respective baselines increases in the order Bezier < cubic spline < linear (with linear baseline being the least accurate and precise).

Even better result regarding the determination of $E$ were obtained by means of the multivariate kinetic analysis, using which the apparent activation energy was determined for each respective sub-process - see upper graphs in Fig. 3. The maximum errors in $E$ values are in these cases approx. $1 \%$ for $E_{S}$ and $0.5 \%$ for $E_{L}$ (again, the linear baseline provides the worst results out of the three tested interpolations). Similar conclusions are valid also for the pre-exponential factor A (which is highly correlated with E); also in case of this quantity the changes are rather negligible $(<1 \%)-$ see the middle row of graphs in Fig. 3. Different situation however arises in case of the model-based quantities. The deviations of the JMA kinetic exponent $\mathrm{n}$ are for the two sub-processes depicted in the lower row of graphs in Fig. 3. None of the tested mathematic interpolations performs particularly well, with the average errors being $\sim 10 \%$. Nonetheless, in certain situations the linear interpolation can result in 
errors as high as $30 \%$. Similarly high level of distortion is present also for the evaluation of the integrated area under the kinetic peak I, with the error magnitude varying around 5 $15 \%$. It is noteworthy that the Bezier interpolation performed particularly well with regard to the determination of the overall area of the kinetic peak (most errors for this interpolation were lower than $5 \%$ ). Significantly worse results were obtained for the ratio between the particular areas attributed to the two overlapping kinetic peaks - this is in Fig. 4 expressed as the relative area corresponding to the smaller kinetic peak $I_{S} / I$ (note that this quantity is very important e.g. for controlled preparation of ceramics/glass-ceramics or for quantitative identification of multicomponent material composition). For the present data the $I_{S} / I$ errors vary between $15-35 \%$, but in one extreme case the $65 \%$ error occurred. Lastly, as the multivariate utilizes non-linear optimization of the whole set of simulated kinetic peaks, the overall correlation coefficient can be obtained as a measure of the quality of the fit; for the sake of clarity the correlation coefficients are for the present data plotted as $\log \left(1-r^{2}\right)-$ see Fig. 4. It is apparent that application of the linear interpolation in case of the downwards step change and data with negative asymmetry (all JMA kinetic peaks exhibit negative asymmetry [37]) results in largest distortions, as demonstrated by almost an order of magnitude worse $\mathrm{r}^{2}$ due to the deviation of the standard JMA model peak shape.

To demonstrate the consequences of the baseline-caused data distortions in kinetic analysis, one needs to realize that one of the ultimate goals of the kinetic analysis are the kinetic predictions. In accordance with this statement, the kinetic results obtained for the most extreme distortions will be compared to the prediction simulated for the original (undistorted) dataset. Simple isothermal prediction (the most common utilization of kinetic calculations) of the conversion change ca. $50^{\circ} \mathrm{C}$ below the onset of the kinetic process measured nonisothermally will be employed for the demonstration - extrapolation by $50{ }^{\circ} \mathrm{C}$ is reasonably large to justify the usefulness of the prediction and, at the same time, to expect an acceptable 
accuracy. The predictions for isothermal annealing at $100{ }^{\circ} \mathrm{C}$ depicted in Fig. 4 are based on the dataset with $A_{L}=10^{14} \mathrm{~s}^{-1}$, as the situation with only partially overlapping processes is more prone to distortions. The largest (percent) errors obtained throughout the present study were employed to show the "worst case scenarios". The black line corresponds to the prediction from the original undistorted data. The solid red line corresponds to the scenario, when only the E and A deviations were taken into account (red solid line; simulation for $E_{S}=151.498 \mathrm{~kJ} \cdot \mathrm{mol}^{-1}, \quad A_{S}=10^{15.163} \mathrm{~s}^{-1}, \quad E_{L}=150.375 \mathrm{~kJ} \cdot \mathrm{mol}^{-1}, \quad A_{L}=10^{14.061} \mathrm{~s}^{-1} ; \quad$ other parameters were similar to the undistorted dataset, i.e. $n_{S}=n_{L}=2, I_{S} / I=0.3$ ). As is apparent, sole deviations of $E$ and $A$ have no effect on the kinetic predictions, even the quite far extrapolated ones. On the other hand, significantly different predictions were obtained when full sets of deviated parameters were taken into account - the graph in Fig. 4 depicts two extreme cases, utilizing the model-based parameters from distorted datasets with $A_{L}=10^{14.5}$ and $A_{L}=10^{15.5}:$ dashed red line $\left(E_{S}=151.498 \mathrm{~kJ} \cdot \mathrm{mol}^{-1}, A_{S}=10^{15.163} \mathrm{~s}^{-1}, E_{L}=150.375 \mathrm{~kJ} \cdot \mathrm{mol}^{-}\right.$ $\left.{ }^{1}, \quad A_{L}=10^{14.061} \mathrm{~s}^{-1}, \quad n_{S}=1.429, \quad n_{L}=2.258, \quad I_{S} / I=0.485\right) \quad$ and dotted red line $\left(E_{S}=151.498 \mathrm{~kJ} \cdot \mathrm{mol}^{-1}, A_{S}=10^{15.163} \mathrm{~s}^{-1}, E_{L}=150.375 \mathrm{~kJ} \cdot \mathrm{mol}^{-1}, A_{L}=10^{14.061} \mathrm{~s}^{-1}, n_{S}=2.531\right.$, $\left.n_{L}=1.717, I_{S} / I=0.202\right)$, respectively. In both these cases it is above all the largely deviated $I_{S} / I$ value that causes the difference in the predictions. Applications-wise, even the most deviated predictions are still quite accurate from the materials stability point of view - these applications are mainly concerned with the low- $\alpha$ (i.e. below 0.1 ) and high- $\alpha$ (above 0.9) predictions, where all the predictions relatively well overlap with the one obtained for the correct set of kinetic parameters. The present distorted predictions would come short only in case of the utilization employing the middle range of $\alpha(0.2-0.8)$, where the predictions can deviate by as much as 0.2 in the degree of conversion - such applications may involve e.g. tailoring of controlled preparation of glass-ceramics or selective preparation of given ratio between the two products corresponding to the two kinetic sub-processes. 


\section{Conclusions}

Theoretical simulations were used to demonstrate the effect of inaccurate baseline interpolations on the results of the consequent analysis of derivative complex kinetic data with different degrees of sub-processes overlaps, where the two sub-processes had similar activation energy. The consequent conclusions were reached:

- general performance of the tested interpolations was (best to worst): Bezier $\rightarrow$ cubic spline $\rightarrow$ linear.

- largest data distortions occur for linear interpolation applied in the only partially overlapped peaks cases when either a downwards heat capacity step accompanies peak with negative asymmetry or an upwards heat capacity step accompanies peak with positive asymmetry.

- isoconversional methods for evaluation of $E$ provided good overall results with errors $<2 \%$ influenced by inaccurate baseline interpolations.

- model-based parameters (kinetic exponents and complexity ratio $I_{i} / I$ ) and integral area of the kinetic peak are significantly influenced by the baseline choice/inaccuracy, the errors in the order of tens of percent occur for these quantities. For model-based complex kinetic studies the tangential area-proportional baseline should therefore strictly be used.

- the choice of baseline (and the associated possible distortions) does not affect the thermal stability predictions for the complex kinetic processes; only the precise predictions based on the mid-range degrees of conversion (e.g. controlled preparation of glass-ceramics) can be significantly affected by the incorrect interpolation of the thermokinetic background.

\section{Acknowledgments}

This work has been supported by the Czech Science Foundation under project No. 17$11753 \mathrm{~S}$. 


\section{References}

[1] Šesták J. Thermophysical Properties of Solids, Their Measurements and Theoretical Analysis. Elsevier: Amsterdam; 1984.

[2] Šesták J. Science of Heat and Thermophysical Studies: A Generalized Approach to Thermal Analysis, Elsevier, Amsterdam, 2005.

[3] H.E. Kissinger, Reaction kinetics in differential thermal analysis, Anal. Chem. 29 (1957) 1702-1706

[4] T. Ozawa, A new method of analyzing thermogravimetric data, Bull. Chem. Soc. Japan 38 (1965) 1881

[5] R.L. Takhor, in: L.L. Hench, S.W. Freiman (Eds.), Advances in Nucleation and Crystallization of Glasses, Amer. Ceram. Soc., Columbus, OH, 1971, pp. 166-172

[6] S. Mahadevan, A. Giridhar, A.K. Singh, Calorimetric measurements on As-Sb-Se glasses, J. Non-Cryst. Solids 88 (1986) 11-34

[7] H.L. Friedman, Kinetics of thermal degradation of char-forming plastics from thermogravimetry. Application to a phenolic plastic, J. Polym. Sci., Part C 6 (1964) 183-195

[8] T. Akahira, T. Sunose, Method of determining activation deterioration constant of electrical insulating materials, Res. Report Chiba Inst. Technol. (Sci. Technol.) 16 (1971) 22-31

[9] M.J. Starink, The determination of activation energy from linear heating rate experiments: a comparison of the accuracy of isoconversion methods, Thermochim. Acta 404 (2003) 163-176

[10] J.H. Flynn, L.A. Wall, General treatment of the thermogravimetry of polymers, J. Res. Nat. Bur. Standards, Part A 70 (1966) 4

[11] S. Vyazovkin, D. Dollimore, Linear and nonlinear procedures in isoconversionalcomputations of the activation energy of thermally induced reactions in solids, J. Chem. Inf. Comp. Sci. 36 (1996) 42-45

[12] S. Vyazovkin, Evaluation of the activation energy of thermally stimulated solidstate reactions under an arbitrary variation of the temperature, J. Comput. Chem. 18 (1997) 393-402

[13] S. Vyazovkin, Modification of the integral isoconversional method to account for variation in the activation energy, J. Comput. Chem. 22 (2001) 178-183

[14] A.I. Lesnikovich, S.V. Levchik, A method of finding invariant values of kinetic parameters, J. Therm. Anal. 27 (1983) 89-93

[15] J. Malek, The kinetic-analysis of nonisothermal data, Thermochim. Acta 200 (1992) 257-269

[16] S. Vyazovkin, A unified approach to kinetic processing of nonisothermal data, Int. J. Chem. Kinet. 28 (1996) 95-101

[17] L.A. Perez-Maqueda, J.M. Criado, P.E. Sanchez-Jimenez, Combined kinetic analysis of solid-state reactions: a powerful tool for the simultaneous determination of kinetic parameters and the kinetic model without previous assumptions on the reaction mechanism, J. Phys. Chem. A 110 (2006) 12456-12462

[18] S. Kitabayashi, N. Koga. Thermal Decomposition of Tin(II) Oxyhydroxide and Subsequent Oxidation in Air: Kinetic Deconvolution of Overlapping Heterogeneous Processes. J. Phys. Chem. C 119 (2015) 16188-16199.

[19] J. Opfermann. Kinetic analysis using multivariate non-linear regression. I. basic concepts. J. Therm. Anal. Calorim. 60 (2000) 641-658 
[20] S. Vyazovkin, A.K. Burnham, J.M. Criado, L.A. Pérez-Maqueda, C. Popescu, N. Sbirrazzuoli, ICTAC Kinetics Committee recommendations for performing kinetic computations on thermal analysis data, Thermochim Acta. 520 (2011) 1-19

[21] S. Vyazovkin, K. Chrissafis, M.L. Di Lorenzo, N. Koga, M. Pijolat, B. Roduit, N. Sbirrazzuoli, J.J. Sunol, ICTAC Kinetics Committee recommendations for collecting experimental thermal analysis data for kinetic computations, Thermochim. Acta 590 (2014) 1-23

[22] R. Svoboda. Linear baseline interpolation for single-process DSC data - yes or no? Thermochimica Acta 655 (2017) 242 - 250

[23] R. Svoboda, J. Málek. Importance of proper baseline identification for the subsequent kinetic analysis of derivative kinetic data, part 1. J. Therm. Anal. Calorim. 124 (2016) $1717-1725$

[24] R. Svoboda. Importance of proper baseline identification for the subsequent kinetic analysis of derivative kinetic data, part 2. J. Therm. Anal. Calorim. 131 (2018) $1889-$ 1897.

[25] J. Šesták, P. Hubík, J.J. Mareš. Hot Topics in Thermal Analysis and Calorimetry Vol.11. Springer: Berlin; 2017.

[26] I.J. Schoenberg. Contributions to the problem of approximation of equidistant data by analytic functions. Part A: On the problem of smoothing of graduation. A first class of analytic approximation formulae. Quart Appl Math. 4 (1946) 45-99

[27] G. Höhne, W. Hemminger, H.J. Flammersheim. Differential Scanning Calorimetry. Berlin: Springer-Verlag; 2003

[28] L. Nowicki, D. Siuta, M. Godala. Determination of the chemical reaction kinetics using isothermal reaction calorimetry supported by measurements of the gas production rate: A case study on the decomposition of formic acid in the heterogeneous Fenton reaction. Thermochimica Acta 653 (2017) 62-70.

[29] M. Catauro, A. Dell'Era, S.V. Ciprioti. Synthesis, structural, spectroscopic and thermoanalytical study of sol-gel derived $\mathrm{SiO} 2-\mathrm{CaO}-\mathrm{P} 2 \mathrm{O} 5$ gel and ceramic materials. Thermochimica Acta 625 (2016) 20-27.

[30] M. Khachani, A. El Hamidi, M. Kacimi, M. Halim, S. Arsalane. Kinetic approach of multi-step thermal decomposition processes of iron(III) phosphate dihydrate FePO4 center dot 2H(2)O. Thermochimica Acta 610 (2015) 29-36.

[31] E.J. Grajales, E.A. Alacron, A.L. Villa. Kinetics of depolymerization of paraformaldehyde obtained by thermogravimetric analysis. Thermochimica Acta 609 (2015) 49-60.

[32] N.N. Begovic, N.N. Stojanovic, S.B. Ostojic, A.M. Radulovic, V.A. Blagojevic, B. Simonovic, D.M. Minic. Thermally induced polymerization of binuclear [Ni$2(\mathrm{en})(2)(\mathrm{H} 2 \mathrm{O})(6)(\mathrm{pyr})]$ center dot 4H(2)O complex. Thermochimica Acta 607 (2015) 82-91.

[33] Johnson WA, Mehl KF. Reaction kinetics in processes of nucleation and growth. Trans Am Inst Min (Metall) Eng. 1939;135:416-42.

[34] Avrami M. Kinetics of phase change I-general theory. J Chem Phys. 1939;7:1103-12.

[35] Avrami M. Kinetics of phase change. II-transformation-time relations for random distribution of nuclei. J Chem Phys. 1940;7:212-24.

[36] Avrami M. Granulation, phase change, and microstructure - kinetics of phase change III. J Chem Phys. 1941;7:177-84.

[37] R. Svoboda, J. Málek. Applicability of Fraser-Suzuki function in kinetic analysis of complex processes. J. Therm. Anal. Calorim. 111 (2013) 1045-56. 


\section{Figure captions}

Fig. 1: A) - D) The four base datasets simulated for different values of $A_{L}$. Each graph depicts set of 7 complex kinetic curves prepared for the heating rates $0.5,1,2,5,10,20$ and $50{ }^{\circ} \mathrm{C} \cdot \mathrm{min}^{-1}$.

E) Demonstration of the construction of the physically meaningful thermokinetic background by using the tangential area-proportional baseline (solid red line) and of its consequent subtraction via the three tested mathematic interpolations. Graph F depicts the zoomed region with baselines.

Fig. 2: Results of the KAS (graphs A and C) and Friedman (graphs B and D) analyses applied to the datasets with different complexity types (located on $\mathrm{X}$ axis) distorted by using the three mathematic interpolations (different types of points) The upper and lower rows of graphs correspond to the averaging of the E- $\alpha$ dependences over the 0.1-0.9 and 0.3-0.7 $\alpha$ intervals, respectively.

Fig. 3: Results of the multivariate kinetic analysis applied to the datasets with different complexity types (located on $\mathrm{X}$ axis) distorted by using the three mathematic interpolations (different types of points). The results for the apparent activation energies $E$, pre-exponential factors $A$ and JMA kinetic exponents $n$ of the smaller (subscript $\mathrm{S}$ ) and larger (subscript L) kinetic sub-processes are shown.

Fig. 4: Results of the multivariate kinetic analysis applied to the datasets with different complexity types (located on $\mathrm{X}$ axis) distorted by using the three mathematic interpolations (different types of points). The results for the overall integrated area $I$, complexity ratio $I_{S} / I$ and correlation coefficient $\mathrm{r}^{2}$ are shown. Graph D depicts the kinetic predictions based on the kinetic parameters obtained from different distorted datasets (red lines) compared to the prediction based on one set of the original undistorted kinetic data $\left(A_{L}=10^{14} \mathrm{~s}^{-1}\right.$, black line $)$ - the predictions were made for the isothermal annealing at $100{ }^{\circ} \mathrm{C}$. 
Fig 1

A

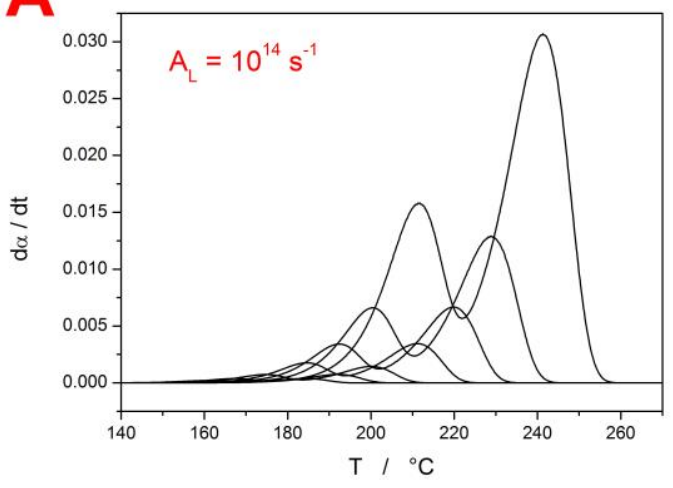

C

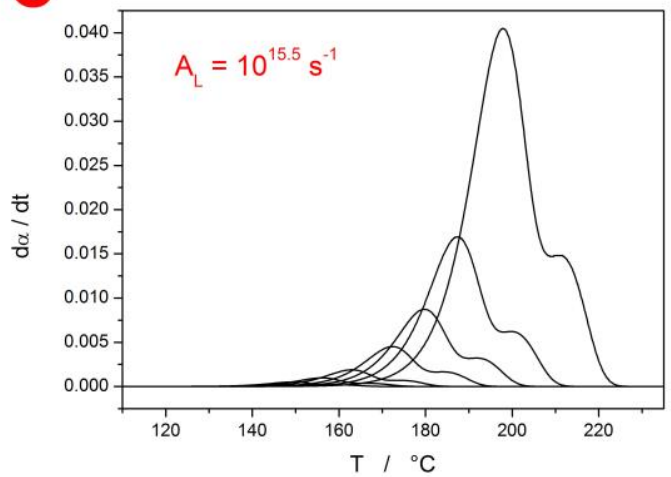

E

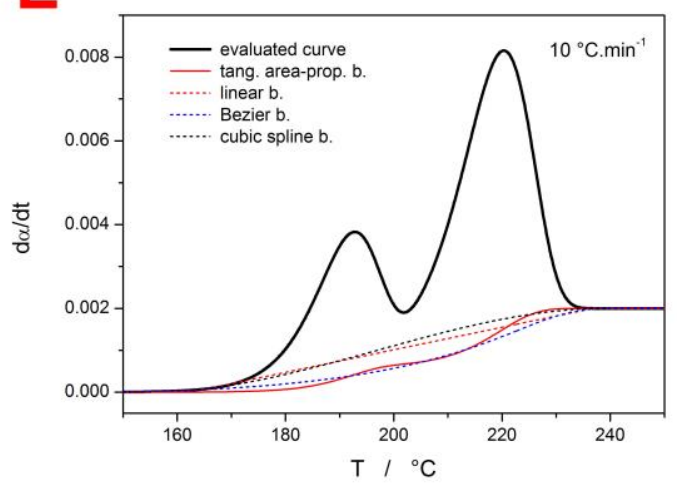

B

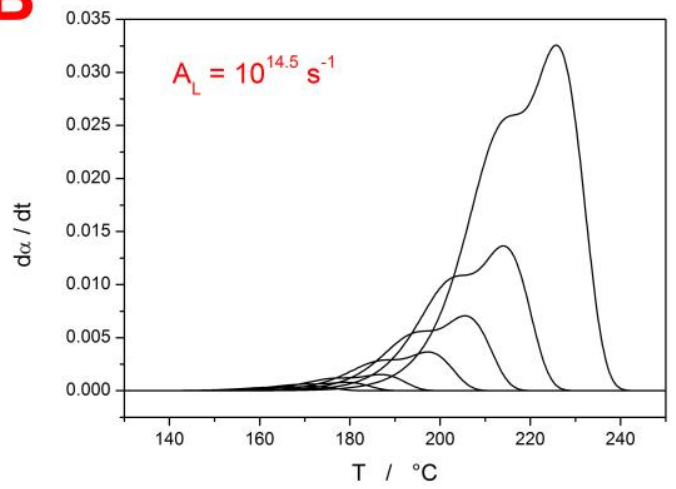

D

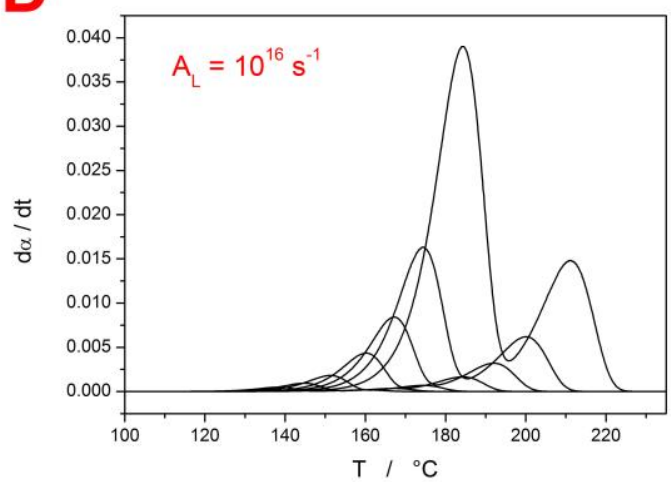

F

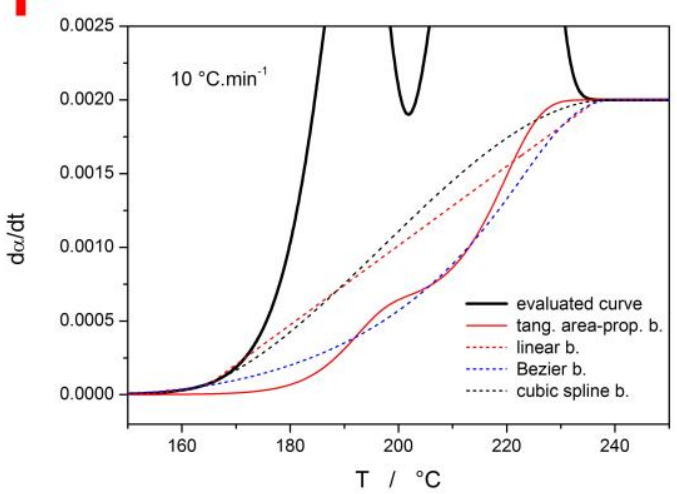


Fig 2
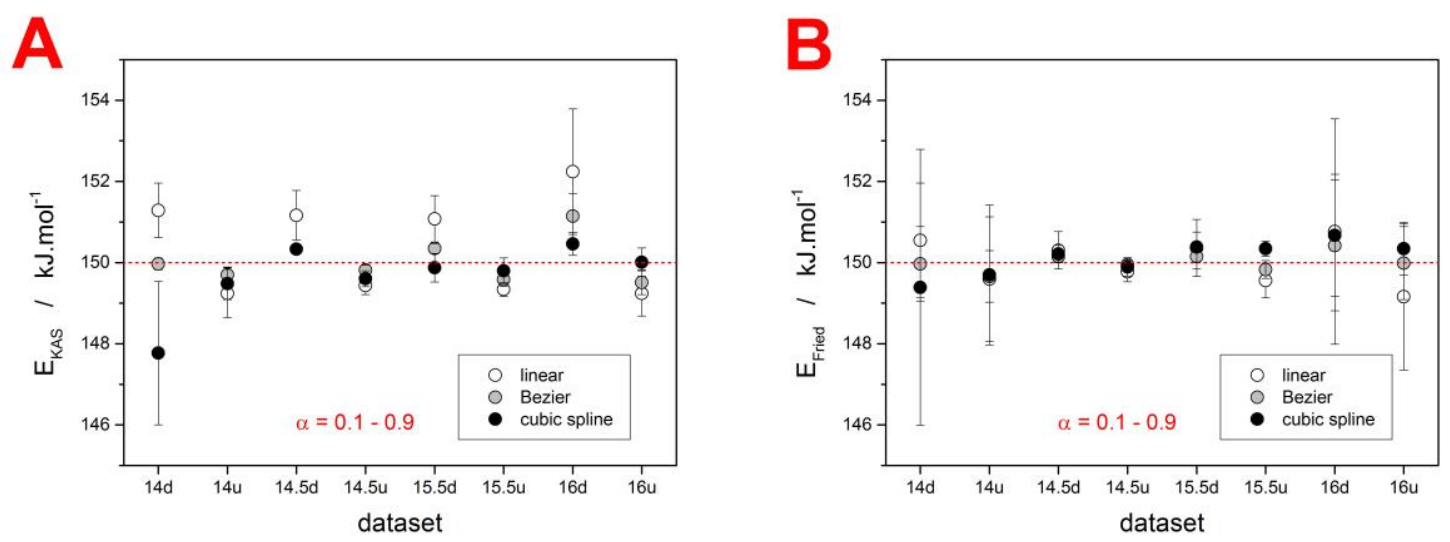

C
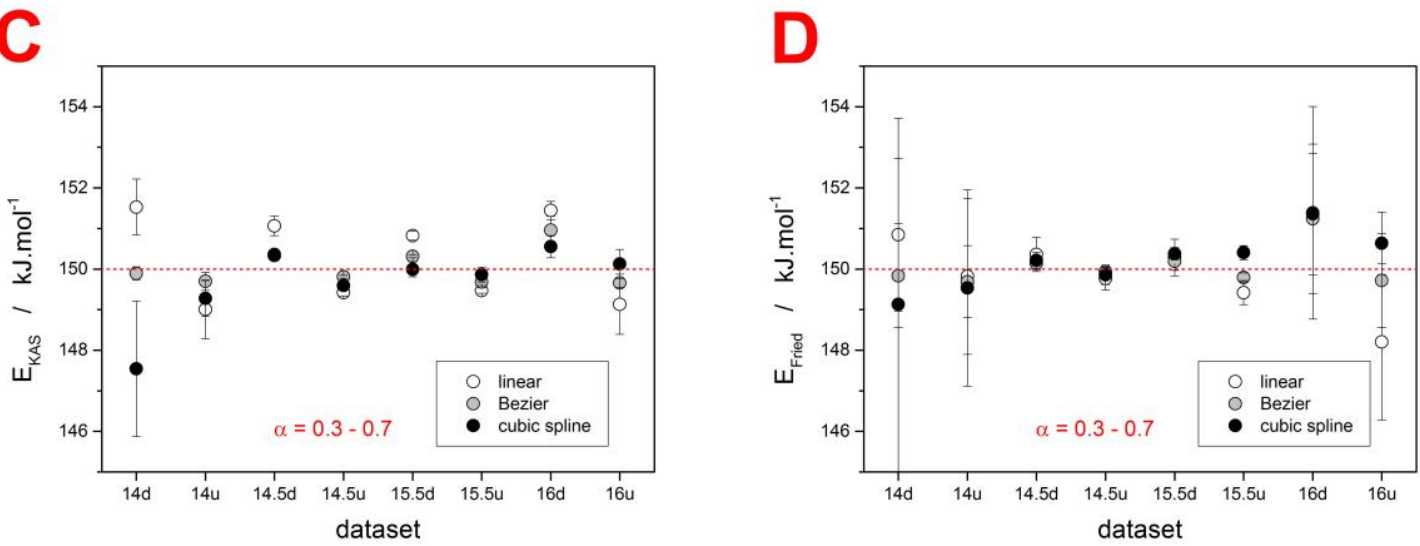
Fig 3
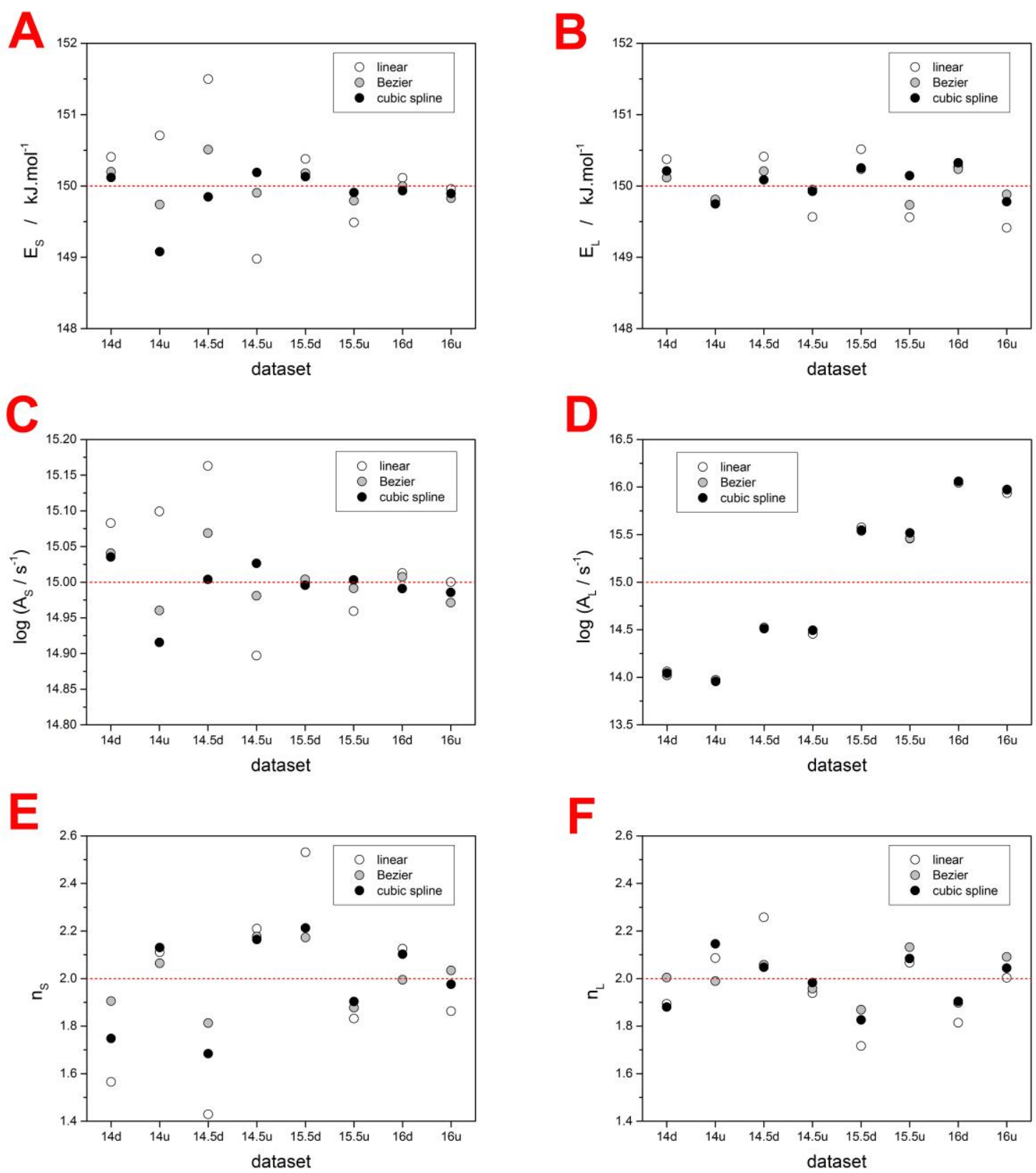
Fig 4

A

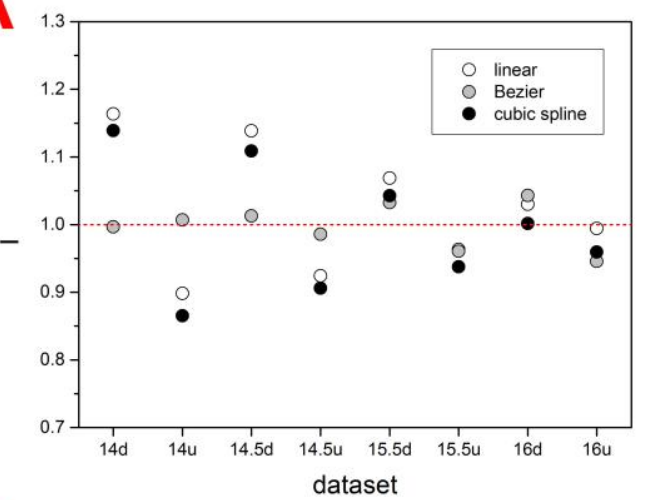

C

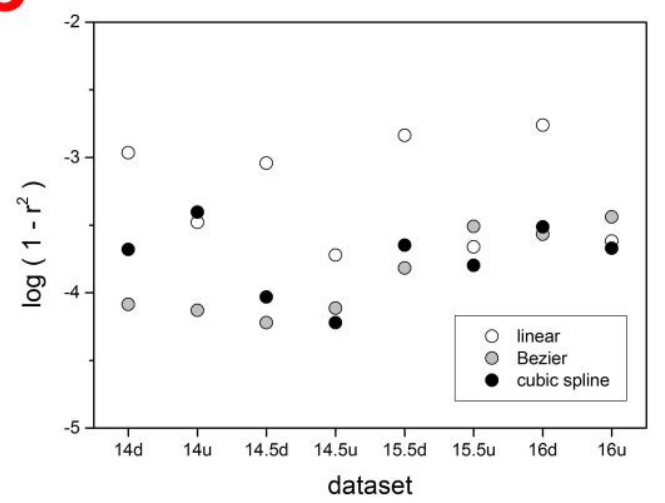

B

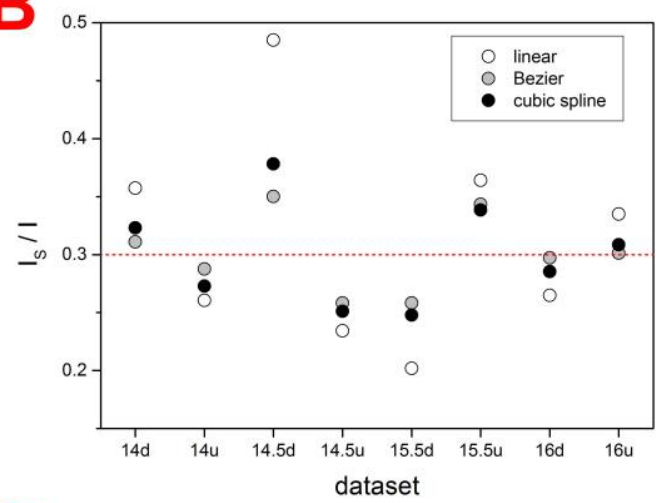

D

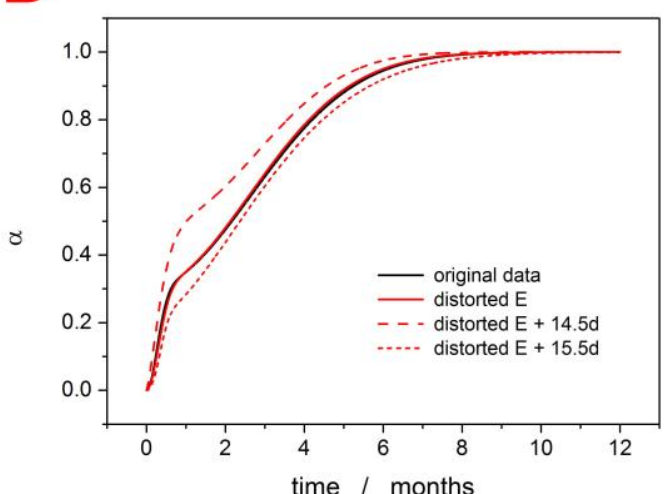

